\title{
Journal review corner - January 2007
}

\author{
Sunil Sharma, Rajeev Srivastava, Kyle Iverson
}

University of South Carolina, West Columbia, USA

Indian J Sleep Med 2006; 1.4, 214-215

\section{Memory Impairment Treated with CPAP}

Memory impairment is a known symptom of obstructive sleep apnea. Now researchers in a recently published study have shown improvement in memory by application of positive airway pressure therapy (CPAP). 58 patients with memory impairment and OSA were treated with CPAP and followed for 3 months. Patients were divided into 1) poor users ( $<2$ hrs./night), moderate users (2-6 hrs.) or optimal users ( $>6$ hrs./ night).

After 3 months patients with optimal use were found to be 8 times as likely to demonstrate normal memory as compared to poor users. This has important clinical implications. Many patients brush of memory loss as part of getting old and there is "nothing you can do about it". However OSA is a reversible and easily treatable cause of memory loss. Secondly, this study again reiterates the importance of compliance and adherence. Patient education is crucial in achieving good results with CPAP therapy.

Zimmerman et al. Chest 2006; 130:1772-1778

\section{Cardiovascular Diseases Associated with Sleep-breathing Disorders}

Sleep-disordered breathing has been associated with development and progression of cardiovascular diseases. Sleep Heart Health Study in which more than 6000

\section{Address for correspondence:}

Sunil Sharma, MD, FCCP, D'ABSM

Director, Columbia Lung \& Sleep Institute

Adjunct Faculty, University of South Carolina

3231 Sunset Blvd, Suite F

West Columbia, SC 29169, USA

Phone : 803-719-5028

Fax: 803-454-2370

E-mail:sunyshar@gmail.com

Sunyshar@yahoo.com

Indian Journal of Sleep Medicine (IJSM), Vol. 1, No. 4, 2006 subjects were screened for sleep-disordered breathing, a significant correlation between RDI and with total serum cholesterol in males and HDL and triglycerides in females was observed.

In a recent study published in European Respiratory Journal an independent relation was found between AHI and HDL but not with total cholesterol, LDL or triglycerides. This effect observed in 366 patients was independent of age, sex, BMI, diabetes or lipid lowering medications. After treating patients with CPAP/BiPAP for 6 months (127 patients) a modest lowering of HDL was observed $(5.8 \%)$.

Since hyperlipidemia is a main risk factor for cardiovascular diseases any therapy that influences it may lead to reduction in cardiovascular morbidity. Results of effect of sleep-disordered breathing on lipid profile have so far been inconsistent. The effects of exercise and fat distribution were not evaluated which may effect HDL levels. However this area needs further investigation to define the association between lipid metabolism and sleep-disordered breathing.

Borgel et al. Eur Respir J 2006; 27:121-127

\section{CPAP Compliance in Patients with Dementia and Alzheimer's}

Compliance and adherence to CPAP therapy has been a major challenge in management of sleep-disordered breathing. Conventional wisdom dictated that this would be even more challenging in patients with dementia. However in a study at University of California, researchers examined whether patients with Alzheimer's disease tolerate CPAP therapy. 30 patients with mild to moderate Alzheimer's disease and OSA were studied. Patients' mini-mental status examination (MMSE) was greater or equal to 18 and $\mathrm{AHI}$ of greater or equal to $10 /$ hr. Patients were then randomized to CPAP or sham CPAP therapy for 3 weeks. Patients were then brought in the lab and group on sham therapy was appropriately titrated and two groups again followed for 3 weeks. 
No significant differences were found between the two groups. Mean CPAP use in the entire group was 4.8 hours. Long-term follow-up up to a year on 21 patients revealed continued adherence in 9 out of 21 patients. Patients with fewer depressive symptoms were found to have better adherence. These results were equivalent to some of the compliance data available in general population. This study suggests that physicians should not be pessimistic when it comes to treating patients with mild to moderate dementia.

Ancoli-Israel et al. Am J Geriatr Psychiatry 2006; 14:176180

\section{Higher Respiratory Diseases Found in Patients With OSAS in Pediatric Population}

The fact that adults with untreated OSA have higher health care utilization is well known. However impact of OSAS in utilization of health care in children has never been examined. Recently researchers looked at 156 children from first year of life to date of diagnosis of OSAS. Children with OSAS were found to have $40 \%$ more hospital visits, $20 \%$ more repeated visits and higher consumption of antibiotics and respiratory drugs. Referral to specialists was also higher. This higher health care utilization and morbidity was mainly due to respiratory tract diseases. This information is beneficial to pediatricians and sleep specialists for early suspicion and intervention.

Tarasuik et al. Am J Respir Crit Care Med Vol 175. pp55-61,2007

\section{Congestive Heart Failure and High Prevalence of Sleep-disordered Breathing}

Patients with congestive heart failure and other conditions causing edema are known to have higher prevalence of sleep-disordered breathing. The reason for this is not well understood. A Study from University of Toronto looks into the possible mechanism of this association. Researchers took 11 healthy subjects and randomized them to either 1) lower body positive pressure with antishock trousers or 2) controls with a crossover design.

The idea was to shift fluid to the neck. Compared with controls, application of anti-shock trousers caused a significant reduction in leg fluid volume and a significant increase in neck circumference and increased airflow resistance of the pharynx. This might contribute to collapse of the nuchal structures. Clinically it reaffirms that aggressive treatment of fluid overload in combination to positive airway pressure in patients with congestive heart failure and sleep-disordered breathing.

Bradley et al. Am J Respir Crit Care Med Vol 174: pp 1378-1383

\section{Playing the Didgeridoo Improves Sleep Apnea}

In a different and interesting approach researchers looked at the effects of playing and practicing didgeridoo (a wind instrument of northern Australia) on snoring and sleep apnea. 25 patients with moderate obstructive sleep apnea were randomized to 1) daily practice of didgeridoo for 25 minutes at home, 6 days a week for 4 months, or 2) control with no intervention.

Compared with the control group in the didgeridoo group daytime sleepiness (Epworth score reduced from average of 11.8 to 7.1 in treatment group and from 11.1 to 9.1 in control group) and apnea-hypopnea index (from average of 22.3 to 11.6 in treatment group and 19.9 to 15.6 in control group) improved significantly and partners reported less sleep disturbance.

The study lends credence to the fact that training of upper airway muscles may play a role in the management of sleep-breathing disorders.

Braendli et al. BMJ 2006;332:266-270

\section{Hypnotic Medications may not improve CPAP compliance}

Patients initiated on CPAP therapy may experience problems with sleep initiation. This may result in poor compliance. Role of short acting hyponotics to improve sleep initiation has not been studied before, though it is not uncommon practice in the clinical world. A recent study looked at the hypothesis that the administration of a hypnotic medication to new CPAP users would facilitate acclimation and increase usage. 72 patients were randomized to 1) oral hypnotic (zolpidem) 2) placebo pill or 3) neither for 14 days at the time of CPAP initiation. Group on oral hypnotics did not show any increase in no of days used or number of hours used/ night as compared to the placebo group or controls. This study does not support the use of hypnotic along with CPAP device to improve compliance. Another danger is that if the patient stops using CPAP device but continues the hyponotics, it may lead to worsening of respiratory events. Hence careful clinical follow-up is mandatory. It is however to be noted that patient selected were not necessarily poor sleepers. It is possible that a sub-set of patients i.e. light sleepers or patients with concomitant Insomnia may have shown better results as many of us have experienced in clinical practice!

Bradshaw et al. Chest Nov;130(5):1369-76

Indian Journal of Sleep Medicine (IJSM), Vol. 1, No. 4, 2006 